\title{
Optical-Theorem-Based Coherent Scatterer Detection in Complex Environments
}

\author{
Edwin A. Marengo and Fred K. Gruber \\ Department of Electrical and Computer Engineering, Northeastern University, Boston, MA 02115, USA \\ Correspondence should be addressed to Edwin A. Marengo; emarengo@ece.neu.edu
}

Received 27 April 2013; Accepted 23 July 2013

Academic Editor: Rocco Pierri

Copyright (C) 2013 E. A. Marengo and F. K. Gruber. This is an open access article distributed under the Creative Commons Attribution License, which permits unrestricted use, distribution, and reproduction in any medium, provided the original work is properly cited.

\begin{abstract}
A new approach is proposed to detect scatterers embedded in reciprocal media from scattering data. The new method is rooted on physical considerations, in particular, on the optical theorem applicable to wavefields (e.g., acoustic, electromagnetic, and optical). The approach exploits insight gained from the optical theorem which assigns physical energy interpretations to the wave data from time reversal mirrors and cavities from which one can deduce the presence of unknown scatterers in unknown background media. The proposed approach is ideally suited for target detection in complex, highly reverberating unknown environments such as indoor facilities, caves, tunnels, and urban canyon.
\end{abstract}

\section{Introduction}

The optical theorem is a well-known result that describes energy conservation in wave scattering phenomena. It defines the rate at which energy is taken away from a probing wavefield by a scattering object, due to both scattering by the object and absorption by the object of part of the incident energy (see [1, page 18], [2, page 716]). The form of this theorem for homogeneous plane wave excitation and freespace background media is well known. In particular, for a scattering potential to the scalar Helmholtz partial differential operator in free space, where the scattering potential or object is interrogated by a time-harmonic homogeneous plane wave, this result states that the rate at which energy is extincted due to scattering and absorption at the scatterer is proportional to the imaginary part of the forward scattering amplitude, corresponding to the direction of propagation of the incident plane wave (see [1], equation 1.80 [2], page 720 [3]). The electromagnetic counterpart is very similar (see [2], page 732 [4]). Recently the theorem has been successfully generalized to arbitrary fields and media, including both reciprocal and nonreciprocal lossless background media [5] (see also the related work in $[3,4]$ ). To facilitate presentation of our ideas, in this paper we focus on reciprocal media. This allows the combination of optical theorem principles with time reversal concepts applicable to reciprocal media (see [6-8] for treatments of time reversal acoustics and electromagnetics).

In this paper we propose a new coherent detection scheme for the acoustic, electromagnetic, or optical detection of unknown scatterers embedded in unknown complex background media. The proposed coherent detection approach is rooted on the optical theorem, which permits the interpretation of scattering data from time reversal mirrors or cavities energetically. A detector of scattering targets, or of changes in a given medium or environment, is proposed that is based on the estimation, from time reversal sensor data, of the total extincted (scattered plus dissipated) power associated with the scattering by the target. In particular, the statistic used for detection is proportional to the total extincted power due to scattering at the target, which in view of the optical theorem can be sensed nonlocally via a time reversal mirror or cavity. This new detection scheme has immediate applications impacting safety and security, for example, as a new sonar- or radar-based approach to the surveillance of indoor facilities, caves, and tunnels and in general complex environments exhibiting significant reverberations that can be exploited via time reversal ideas. In this work we discuss the physical detection principles behind this new approach to detecting unknown targets in unknown 
media, and propose two variants of a new optical-theorembased coherent detector. The statistical performance of the new detection approach is examined for additive Gaussian noise in the rigorous framework of detection theory. The new detection scheme is compared with the alternative approach called "energy detection" which is customarily used in the detection of unknown signals [9]. Two major differences between our new approach and the conventional approach are emphasized throughout the paper: (1) that while the energy detector is based on a "mathematical energy" or $L^{2}$ norm, our new detector is based on real physical energy or power; and (2) that while the energy detector does not use phase information and is then "incoherent," our new approach exploits phase information and is "coherent." The analytical and numerical results presented in the paper shed light on the practical applicability of the proposed approach and illustrate its advantages over the conventional energy detection scheme.

The paper is organized as follows. In Section 2 we present the key physical principles of the new detection approach. In Section 3 we establish the detailed detection-theoretic analysis of both the conventional energy detector and the new coherent detector approach. In Section 4 we provide numerical validation of the ideas and results discussed in the paper. Section 5 provides concluding remarks.

\section{Physical Detection Principles}

Consider, under suppressed time-harmonic dependence, the active detection of a scatterer located in a region of investigation $V$. The scatterer is embedded in a lossless wave propagation medium that can be bounded or unbounded, and this is incorporated into the model via the suitable boundary conditions. Our discussion applies, in particular, to two possible sensing modalities: electromagnetic (e.g., microwave) systems described by Maxwell's equations, as well as generally nonhomogeneous but lossless acoustic systems describable in the frequency domain by the basic scalar Helmholtz equation

$$
\left[\nabla^{2}+k^{2}(\mathbf{r})\right] \psi(\mathbf{r})=\rho(\mathbf{r}),
$$

where $\psi$ is the pressure wavefield, $\rho$ is the source that radiates the field, and the real-valued $k(\mathbf{r})$ is the wavenumber of the field at the given frequency and position $\mathbf{r}$. The model in (1) is also relevant to high-frequency (e.g., optical) systems.

The datum corresponding to a scattering experiment $(m, n)$ is defined by (1) a known acoustic $\left(\rho_{n}\right)$ or electromagnetic $\left(\mathbf{J}_{n}, \mathbf{M}_{n}\right)$ source, where $n$ denotes the transmitter state, for example, its position, orientation, and so forth, that is located outside $V$ and radiates in the background medium a given incident acoustic $\left(\psi_{n}^{(i)}\right)$ or electromagnetic $\left(\mathbf{E}_{n}^{(i)}, \mathbf{H}_{n}^{(i)}\right)$ field; and (2) a receiver outside $V$ whose output $v_{m, n}$ is a linear projection of the scattered acoustic $\left(\psi_{n}^{(s)}\right)$ or electromagnetic $\left(\mathbf{E}_{n}^{(s)}, \mathbf{H}_{n}^{(s)}\right)$ field, onto a given state $m$ of the acoustic $\left(I_{m}\right)$ or electromagnetic $\left(\mathbf{I}_{m}, \mathbf{K}_{m}\right)$ form, in particular, for acoustic sensing

$$
v_{m, n}=\int_{\mathbf{r} \notin V} d \mathbf{r} I_{m}(\mathbf{r}) \psi_{n}^{(s)}(\mathbf{r})
$$

and for electromagnetic sensing

$$
v_{m, n}=\int_{\mathbf{r} \notin V} d \mathbf{r}\left(\mathbf{I}_{m} \cdot \mathbf{E}_{n}^{(s)}-\mathbf{K}_{m} \cdot \mathbf{H}_{n}^{(s)}\right) .
$$

In view of the reciprocity principle, the acoustic $I_{m}$ or electromagnetic $\mathbf{I}_{m}, \mathbf{K}_{m}$ are the sources or sinks representing the receiving transducer or antenna, while the scattering datum $v_{m, n}$ has the meaning of a reaction. If these $m$ labelled sources are chosen such that they radiate, in the reciprocal background medium, fields whose values within $V$ are equal to those of the complex conjugate fields $\left(\psi_{m}^{(i) *}\right.$ in the acoustic case or $\mathbf{E}_{m}^{(i) *},-\mathbf{H}_{m}^{(i) *}$ in the electromagnetic case) corresponding to $m$-labelled sources ( $\rho_{m}$ in the acoustic case or $\mathbf{J}_{m}, \mathbf{M}_{m}$ in the electromagnetic case), then the datum $v_{m, n}$ has the simultaneous meaning of a reaction and an energy interaction, and, in particular, it carries information about the power budget of a well-defined scattering experiment which can involve one or two sources (one if $m=n$ and two if $m \neq n$ ) [5]. The relation describing the power budget in question is called the generalized optical theorem. For the particular case $m=n$ this general result takes the following form, called the ordinary optical theorem:

$$
\frac{1}{2} \Re\left(v_{n, n}\right)=P_{n}^{(s)}+P_{n}^{(\text {loss })},
$$

where $\Re$ is the real part, $P_{n}^{(s)}$ is the total scattered power, and $P_{n}^{\text {(loss) }}$ is the power dissipated (as heat) inside the scatterer, upon excitation by the $n$-labelled incident field. Furthermore, within the scalar treatment and in the electromagnetic case for nonmagnetic scatterers, the quantity $(1 / 2) \mathfrak{J}\left(v_{n, n}\right)$ corresponds to the reactive power resulting from field energy storage in the near field of the scatterers (see Section $\mathrm{V}$ of [5]). Thus the magnitude $(1 / 2)\left|v_{n, n}\right|$ represents the total apparent power of the scattering phenomenon, and any of these quantities $\left(\mathfrak{R}\left(v_{n, n}\right), \mathfrak{I}\left(v_{n, n}\right),\left|v_{n, n}\right|\right)$ can be used as the physical basis of a test statistic for detection. In this paper we discuss the use of test statistics based on (4) and $\left|v_{n, n}\right|$ paying particular attention to the latter since it carries information about both (far-field) scattering and (near-field) energy storage due to the presence of the target.

In particular, for reciprocal backgrounds, for which the ideas of time reversal focusing can be applied, the optical theorem can be applied to detect targets in a region $V$ as follows. Consider a background medium such as the indoor environment shown in Figure 1. A transmitter located outside $V$ is used to interrogate the scatterer. An array of receivers which will act in the following as the detector is placed outside $V$. Two situations are possible: (1) there is no target at the beginning; (2) or there is a target but the target changes (e.g., the target may be a person hiding in a facility, who is not moving originally but begins to walk at a subsequent time). In the first case the goal is to detect the presence of the 


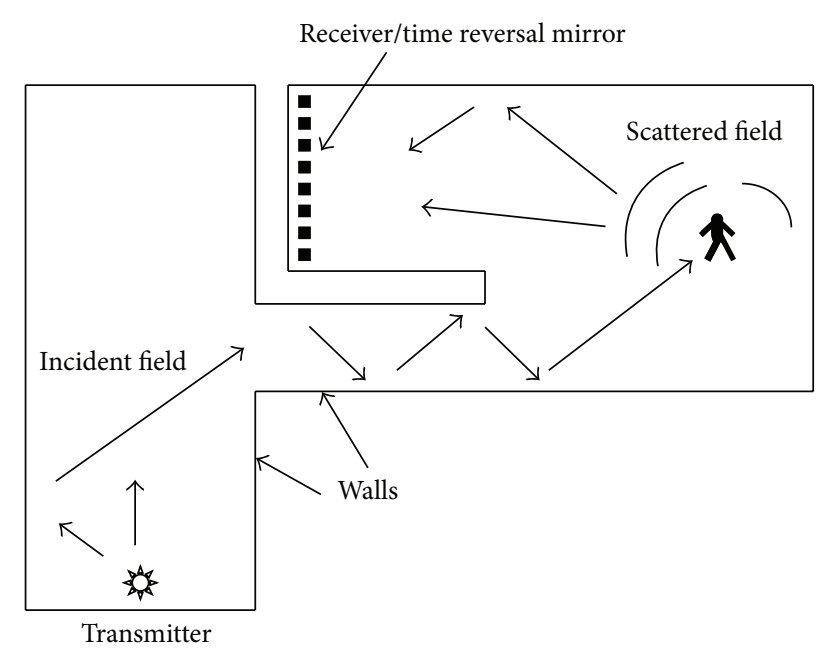

Figure 1: Optical-theorem-based target detection in an indoor environment.

target, while in the second case we want to detect the target change, for example, the target moves to a different position. To facilitate exposition, in the following we emphasize the first situation only; however, it is easy to apply the same general concept to the second situation. The scattering data are gathered as follows.

(1) Transmission: the transmitter radiates in the absence of the target given incident fields $\left(\psi_{n}^{(i)}\right.$ in the acoustic case or $\mathbf{E}_{n}^{(i)}, \mathbf{H}_{n}^{(i)}$ in the electromagnetic case).

(2) Reception: the receiver array locally senses this incident field due to the transmitter.

(3) Define the time-reversal-based filter: a source that produces in $V$ a field that is approximately equal to the time-reversed version of the incident field in $V$ produced in step 1 is the familiar time reversal mirror obtained by driving the array elements with the timereversed version of the received signals (scaled by a multiplicative constant); therefore, in this step we choose the filtering or weights of the receiver array elements precisely as the vector signal corresponding to the time-reversed version of the signals received in step 2.

(4) Process the scattered field signal with the timereversal-based filter: if the field produced by the timereversal mirror approximates well the time-reversed version of the incident field of step 1 inside the region of investigation $V$, then the output $f_{n}$ of the filtering of the scattered field by this filter is approximately equal to $v_{n, n}$ multiplied by a constant, so that according to (4) it is directly related to the total power (both scattered and dissipated) that is extincted by the scattering target. Note that the processing is of the scattered field signal, so that to the output signal measured at the array one must subtract the filtered output without the scatterer.
(5) Decision: if $\Re\left(f_{n}\right)$ is above a threshold $t$ that depends on the noise level, then it seems from (4) that real power is taken away from the probing field (extincted) which indicates the presence of a target in $V$; then we choose the "target present" alternative, while if $\Re\left(f_{n}\right)<t$ we choose "no target present." Alternatively, we can use the apparent power statistic, $\left|f_{n}\right|$, so that if $\left|f_{n}\right|>t$ then we choose the "target present" alternative while if $\left|f_{n}\right|<t$ we decide that there is "no target present." This physical description will be formalized in detection theoretical terms in the following section.

To fix ideas, consider, for example, scalar wavefield signals relevant to acoustic or optical sensing scenarios. Let $\psi_{n}^{(i)}(\mathbf{r})$ be the incident scalar signal of the $n$th transmitter (the $n$ may represent a given transmitter state; e.g., if the transmitter changes position it can represent the $n$th position). The incident field signal at an $M$-element receiver array with positions $\mathbf{R}_{1}, \ldots, \mathbf{R}_{M}$ is then

$$
p_{i, n}=\left[\psi_{n}^{(i)}\left(\mathbf{R}_{1}\right), \ldots, \psi_{n}^{(i)}\left(\mathbf{R}_{M}\right)\right]^{T},
$$

where $T$ denotes the transpose. The time-reversal-based filter is then

$$
p_{i, n}^{*}=\left[\psi_{n}^{(i) *}\left(\mathbf{R}_{1}\right), \ldots, \psi_{n}^{(i) *}\left(\mathbf{R}_{M}\right)\right]^{T} .
$$

The output corresponding to the incident field signal alone is then

$$
\left\|p_{i, n}\right\|^{2}=\sum_{m=1}^{M}\left|\psi_{n}^{(i)}\left(\mathbf{R}_{m}\right)\right|^{2}
$$

The scattered field $\psi_{n}^{(s)}$ is equal to the total field $\psi_{n}$ in the presence of the target minus the incident field $\psi_{n}^{(i)}$; thus the output associated to step 4 above is

$$
\begin{aligned}
f_{n} & =p_{i, n}^{H} p_{s, n} \\
& =\sum_{m=1}^{M} \psi_{n}^{(i) *}\left(\mathbf{R}_{m}\right) \psi_{n}^{(s)}\left(\mathbf{R}_{m}\right)-\left|\psi_{n}^{(i)}\left(\mathbf{R}_{m}\right)\right|^{2} \propto v_{n, n},
\end{aligned}
$$

where $H$ denotes the complex conjugate transpose and

$$
p_{s, n}=\left[\psi_{n}^{(s)}\left(\mathbf{R}_{1}\right), \ldots, \psi_{n}^{(s)}\left(\mathbf{R}_{M}\right)\right]^{T} .
$$

Note that this corresponds to a matched filter based on the incident signal measured at the receiver. Detection algorithms based on (8), for example, "target present" if $\left|f_{n}\right|>t$ or "no target present" if $\left|f_{n}\right|<t$, work so long as the time reversal mirror (the receiver-turned-transmitter array driven by the excitations in (6)) effectively reproduces the time-reversed incident fields in the region where the target is located. Due to reciprocity, the roles of the transmitter and receiver can be interchanged, with the mirror acting as the transmitter, which is relevant to the military and security scenario sketched earlier. Note that (8) represents coherent processing, using the scattered-field phase information. It contrasts with the 
alternative scheme based on the 2 norm of the scattered signal which is incoherent. The coherent approach uses a statistic that is related to the global power budget information about the scatterer while the incoherent approach is based on a statistic corresponding to a mathematical energy, that is only approximately related to the local scattered power density reaching the receiver aperture.

Importantly, the proposed detector does not assume knowledge of the position of the transmitter or the details of the medium other than it is sufficiently reverberating to allow synthesis of the desired complex conjugate field via a time reversal mirror (more generally one may need a time reversal cavity). This is very important for practical applications such as military and security scenarios. For example, an officer carrying the transmitter may enter the facility, tunnel, and so forth, while a separate detector array senses the signals and makes decisions that can be sent wirelessly to the officers in the scene regarding the presence or absence of another person in the facility, and so forth. In another, biomedical scenario, one may temporarily place transmitters inside the patient (e.g., via the digestive track), while the detector array remains outside, to detect, for example, changes in a tumor after treatment or the ingestion of a biomarker aimed at detecting the tumor. It is only assumed that the array of receivers represents a sufficiently large aperture to allow the radiation inside $V$ of the complex conjugate or time-reversed version of the fields due to the transmitter. In highly reverberating media, realistically sized time reversal mirrors can be used to produce fields that are good approximations in the interior of that medium of the time-reversed fields associated with realistically sized transmitters.

\section{Detection Theory Results}

In the rest of the paper we suppress the implicit $n$ dependence with the understanding that the results hold for an $n$th experiment or snapshot, corresponding to an $n$th transmitter state. In addition, so far we have outlined the physical principles motivating a new coherent detection approach, but up to this point no concrete reference to a particular statistical signal processing or noise model has been considered. In this section we take explicit account of the realistic noise and develop the formal detection theoretic analysis quantifying the performance of the optical-theorem-based coherent detector for unknown scatterers in unknown media (see [10] for an overview of the basic detection theory concepts).

We assume that the incident field $\psi^{(i)}$ corresponding to the field generated in the medium by the excitation source without the target is measured at the receiver array and is therefore known in the signal processing that follows. This field is measured at the locations of the receiver array elements, $\mathbf{R}_{m}, m=1, \ldots, M$. Then the respective $M \times 1$ incident field vector is

$$
p_{i}=\left[\psi^{(i)}\left(\mathbf{R}_{1}\right), \ldots, \psi^{(i)}\left(\mathbf{R}_{M}\right)\right]^{T} .
$$

We emphasize that the medium itself remains unknown. All that is known is the locally measured incident field vector $p_{i}$. For simplicity, we assume in the following that the measurement of $p_{i}$ is very accurate (we will, however, explore the effect of realistic noise in the incident field with numerical simulations in Section 4). On the other hand, we attach realistic sensor noise to the total received signal and associated measured scattered field signal, as follows.

In particular, if at a later time a target appears in this medium, then the vector signal sensed at the receiver array is

$$
p_{t}=\left[\psi\left(\mathbf{R}_{1}\right), \ldots, \psi\left(\mathbf{R}_{M}\right)\right]^{T}
$$

where the total field in the presence of the target is

$$
\psi(\mathbf{r})=\psi^{(s)}(\mathbf{r})+\psi^{(i)}(\mathbf{r})
$$

where $\psi_{s}$ is the scattered field due to the target. Thus the respective scattered field vector,

$$
p_{s}=\left[\psi^{(s)}\left(\mathbf{R}_{1}\right), \ldots, \psi^{(s)}\left(\mathbf{R}_{M}\right)\right]^{T},
$$

is given from (10), (11), and (12) by

$$
p_{s}=p_{t}-p_{i}
$$

In practice the received signal $p_{t}$ exhibits noise. We consider the basic signal model

$$
\widehat{p}_{t}=p_{t}+w
$$

where $w$ is circular complex Gaussian noise, in particular,

$$
w \sim \mathscr{C} \cdot \mathcal{N}\left(0, \sigma^{2} \mathbf{I}_{M}\right),
$$

where $\mathbf{I}_{M}$ is the $M \times M$ identity matrix. Then also the measured scattered field signal is corrupted by noise, and (14) takes in view of (15) with the substitution $p_{t} \rightarrow \widehat{p}_{t}$ the realistic form

$$
y=\widehat{p}_{t}-p_{i}=p_{s}+w
$$

where to keep the notation simple we use $y$ to denote the noisy scattered field measurement. Then $y \sim \mathscr{C} \mathscr{N}\left(p_{s}, \sigma^{2} \mathbf{I}\right)$. Therefore the conditional probability distribution functions (pdfs) for the two hypotheses $\left(H_{0}\right.$ : "no scatterer present"; $H_{1}$ : "scatterer present") are as follows:

$$
\begin{gathered}
p\left(y \mid H_{0}\right)=\frac{1}{\pi^{M}\left(\sigma^{2}\right)^{M}} \exp \left(\frac{-\|y\|^{2}}{\sigma^{2}}\right) \\
p\left(y \mid H_{1}\right)=\frac{1}{\pi^{M}\left(\sigma^{2}\right)^{M}} \exp \left(\frac{-\left\|y-p_{s}\right\|^{2}}{\sigma^{2}}\right) .
\end{gathered}
$$

3.1. Energy Detector. In detecting unknown signals, such as the scattered signals considered in this paper, it is customary to employ an incoherent detection scheme termed "the energy detector" [9]. The energy detector uses as test statistic the mathematical signal energy or $L^{2}$ norm of the measured signal. In the present scatterer detection context the relevant 
signal is the measured scattered field vector $y$, so that the signal energy is

$$
E(y)=\|y\|^{2}=\sum_{m=1}^{M}|y(m)|^{2}
$$

For convenience, we adopt the normalized quantity

$$
\bar{E}=\frac{2 E}{\sigma^{2}}
$$

The detector works by comparing the value of $\bar{E}$ relative to a threshold $t$. If $\bar{E}>t$ the decision "target present" is made while if $\bar{E}<t$ the decision "no target present" is made.

Since the signal in the absence of the scatterer, $\left(y \mid H_{0}\right) \sim$ $\mathscr{C} \mathcal{N}\left(0, \sigma^{2} \mathbf{I}\right)$ (noise only), while the signal in the presence of the scatterer, $\left(y \mid H_{1}\right) \sim \mathscr{C} \mathscr{N}\left(p_{s}, \sigma^{2} \mathbf{I}\right)$, then the respective conditional pdfs of the test statistic equation (20) are as follows:

$$
\begin{aligned}
& \bar{E}\left(y \mid H_{0}\right) \sim \chi_{2 M}^{2}(0), \\
& \bar{E}\left(y \mid H_{1}\right) \sim \chi_{2 M}^{2}(\mu),
\end{aligned}
$$

where

$$
\mu=\frac{2\left\|p_{s}\right\|^{2}}{\sigma^{2}} .
$$

Therefore the probability of detection, $P_{d}=P$ ("target present" $\left.\mid H_{1}\right)$, is given by

$$
P_{d}=1-\Psi_{2 M, \mu}(t)
$$

where $\Psi_{2 M, v}(x)$ denotes the cumulative distribution function (cdf) of a noncentral $\chi$-square random variable with $2 M$ degrees of freedom and noncentrality parameter $\nu$. Similarly, the probability of false alarm, $P_{\mathrm{fa}}=P$ (“target present" $\mid H_{0}$ ), is given by

$$
P_{\mathrm{fa}}=1-\Psi_{2 M, 0}(t)
$$

3.2. New Coherent Scatterer Detector. We discussed in Section 2 that an important consequence of the optical theorem is that in complex, ideally highly reverberating media, the real part of the quantity

$$
f=p_{i}^{H} p_{s}
$$

(where $H$ denotes complex conjugate transpose) carries information about the total scattered power due to the target. Furthermore, the imaginary part of this quantity carries the complementary total reactive power information [5]. Thus the magnitude of this quantity is a measure of the total apparent power associated with the scattering. Therefore it

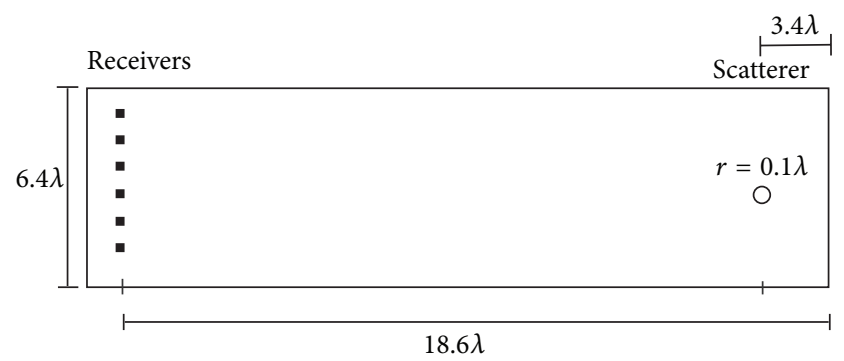

FIGURE 2: Scatterer detection in a waveguide of perfectly reflecting walls.

is logical to formulate the scatterer detection problem in complex media using as test statistic the amplitude

$$
f_{\mathrm{Re}}=\Re\left(p_{i}^{H} y\right)
$$

or alternatively the apparent power version

$$
f^{\prime}(y)=\left|p_{i}^{H} y\right| \text {. }
$$

For example, in the apparent power approach (27), if $f^{\prime}(y)>$ $t$ where $t$ is a detection threshold, then we declare "target present," while if $f^{\prime}(y)<t$ we choose "no target present."

It is important to emphasize the fundamental differences between these two approaches, coherent (based on (26), and (27)) versus incoherent (based on (20)), to scatterer detection of unknown scatterers in unknown complex media. First, the test statistic in the incoherent approach throws away the phase information of the measured scattered signal. Only the scattered signal amplitude (of each entry $y(m)$ ) is utilized in the decision making. This contrasts with the new coherent approach which is based on matched filtering based on the incident field signal $p_{i}$ that was measured in the absence of the scatterer as part of a prior, "no-target-present" sensing step. In this coherent processing both the amplitude and phase of the scattered signal are taken into account. Second, in the conventional incoherent detection approach one uses the key incident field signal $p_{i}$ only in the background suppression step equation (14) leading to (17) and disregards this signal afterwards. In contrast, the coherent detection approach of this work makes use of the incident field signal $p_{i}$ twice: first in the elemental background suppression step and next as the key reference signal in the subsequent matched filter detector which is based on energy conservation considerations applicable to highly reverberating media. In the coherent approach, $p_{i}$ is understood to carry valuable information about the unknown complex medium itself, to the point that it is in fact the key signature in the matched filtering adopted for detection in that complex medium. It is also important to point out that the matched filter in (26) and (27) is not the familiar matched filter that is customarily used in standard signal processing and detection which employs the known information-carrying signal that is added to the noise. In the present case we assume no knowledge whatsoever either of the propagation medium or of the scatterer, so we do not know the scattered signal. Therefore we cannot implement the standard matched filter approach. 


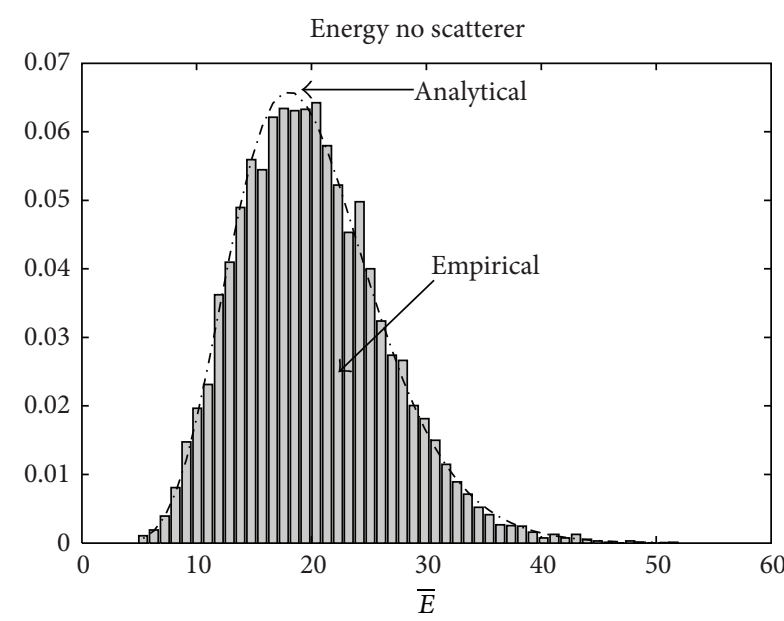

(a)

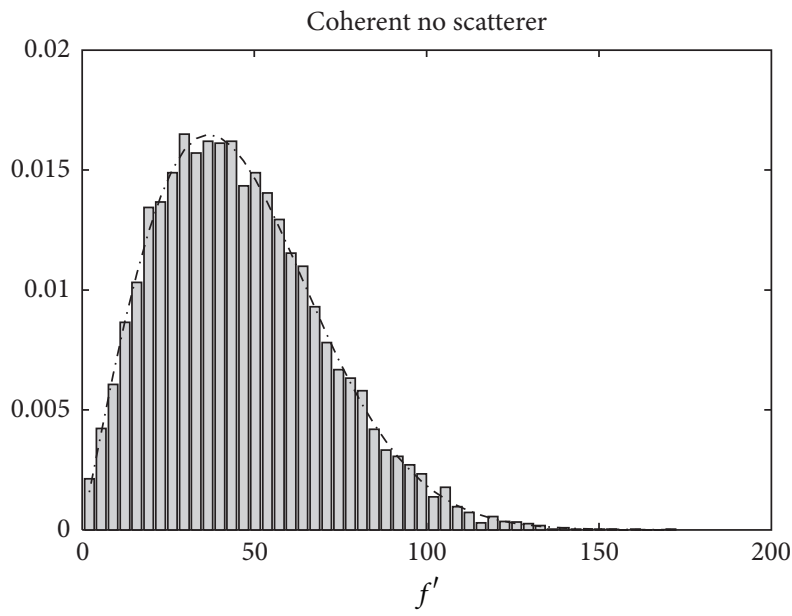

(c)

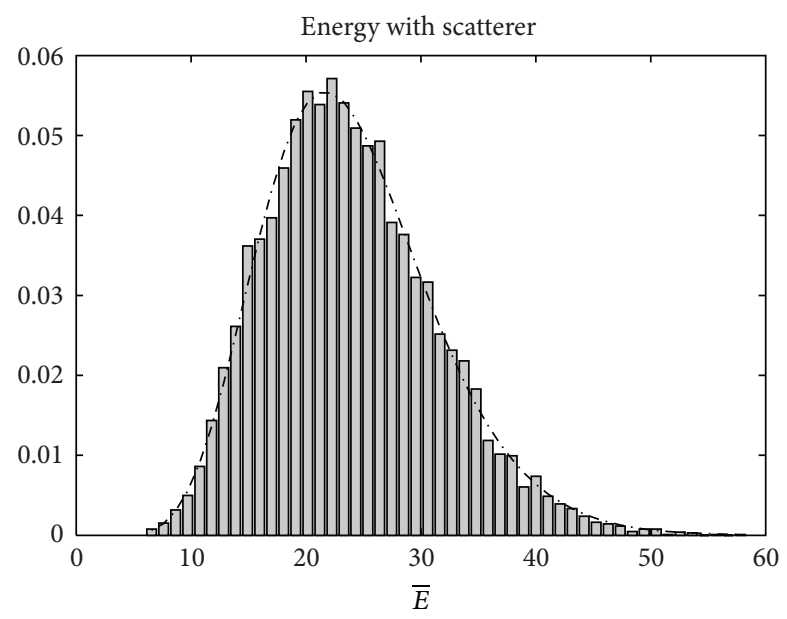

(b)

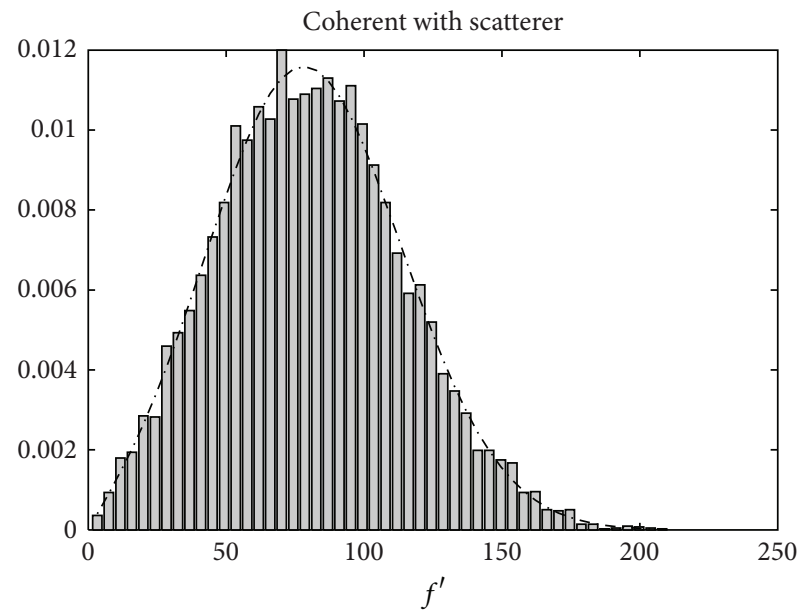

(d)

FIGURE 3: Histograms for scatterer detection in a waveguide of perfectly reflecting walls.

In particular, the standard matched filter counterpart of our approach equation (26) is given by

$$
g=\mathfrak{R}\left(p_{s}^{H} y\right)
$$

which is equivalent to the well-known likelihood ratio test statistic if $p_{s}$ is known, but we do not know $p_{s}$ and therefore cannot use this approach. Thus, our "optical theorem matched filter" in equations (26) and (27) is of an entirely different, physical nature. It is rooted on physical considerations that cannot be derived from the signal model alone without reference to the wave propagation physics that describes the physical process giving rise to the measured signals. In particular, the energy in the conventional energy detector is mathematical energy, while the real and apparent power discussed in (25), respectively, (and in the noisy counterparts equations (26) and (27)) is a real, measurable, physical quantity. Based on this phenomenological insight, we anticipate that the proposed coherent scatterer detector may outperform the energy detector in many scenarios, particularly in environments that are highly reverberating so that the well-known principles of time reversal acoustics and electromagnetics can be applied successfully.

We elaborate next the probabilistic performance of the proposed coherent detector based on the optical theorem matched filter in (26) and (27). It is easy to show that

$$
f_{\operatorname{Re}}\left(y \mid H_{0}\right) \sim \mathcal{N}(0, \zeta),
$$

where

$$
\zeta=\frac{\left\|p_{i}\right\|^{2} \sigma^{2}}{2}
$$

while

$$
f_{\mathrm{Re}}\left(y \mid H_{1}\right) \sim \mathcal{N}\left[\Re\left(p_{i}^{H} p_{s}\right), \zeta\right] .
$$

From these results and the cdf of the normal distribution it is easy to compute $P_{d}$ and $P_{\mathrm{fa}}$ for the detector in (26) in a way analogous to (23) and (24). In the rest of the paper we focus on 


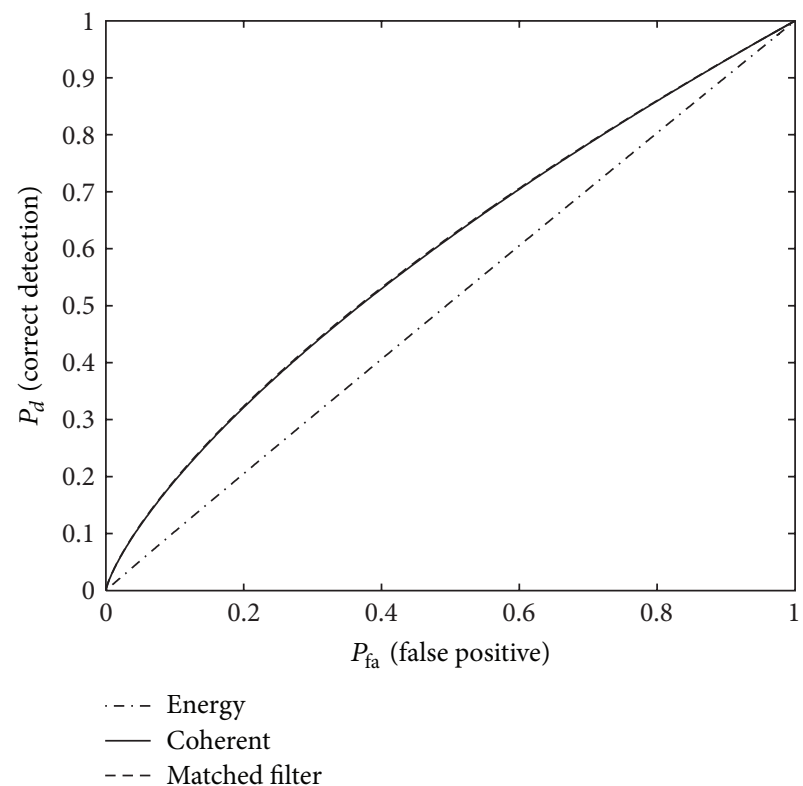

(a) ROC curve with 2 receivers

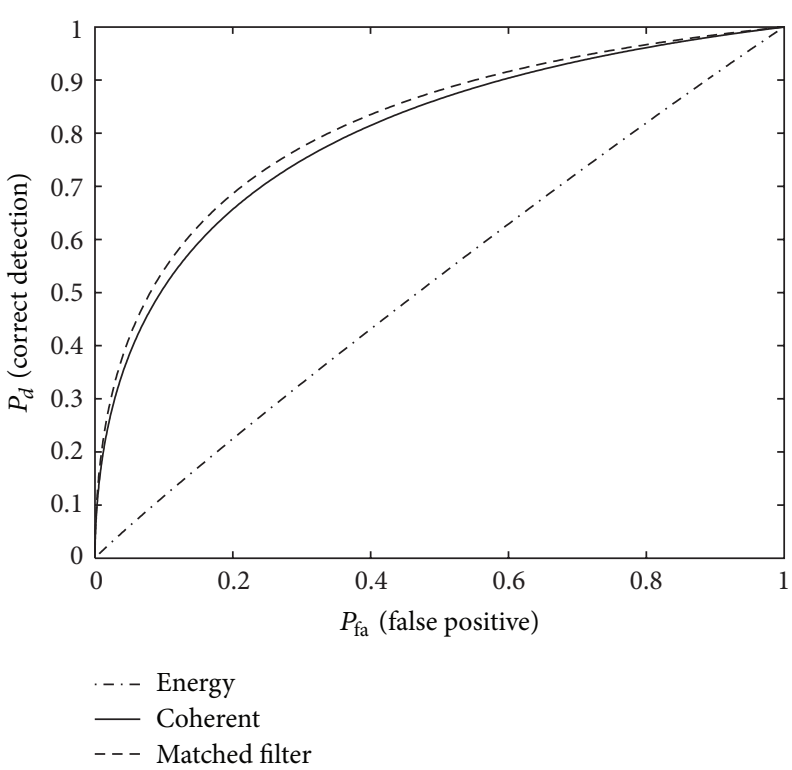

(c) ROC curve with 10 receivers

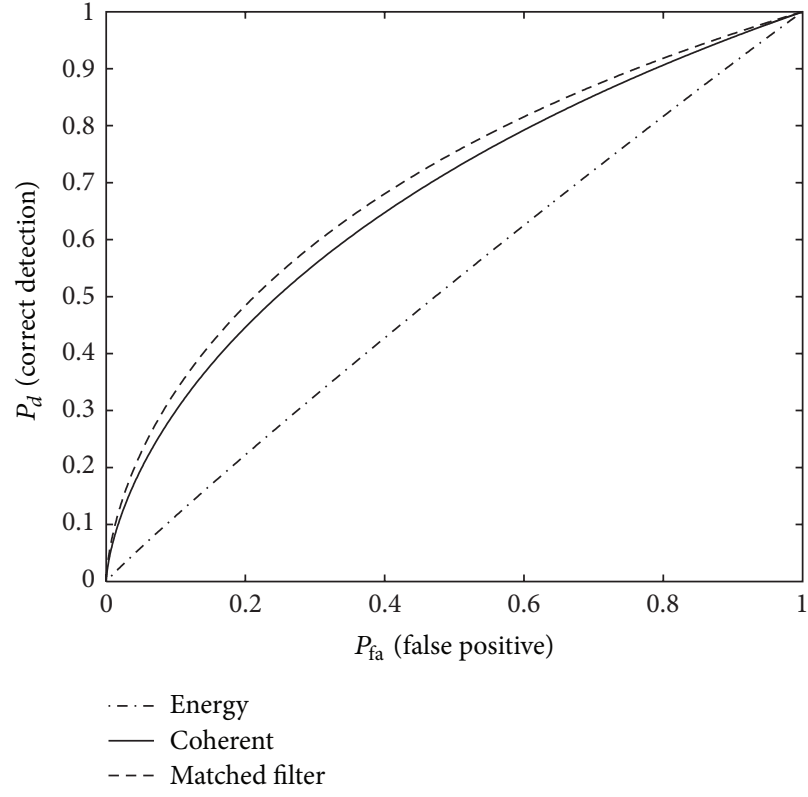

(b) ROC curve with 5 receivers

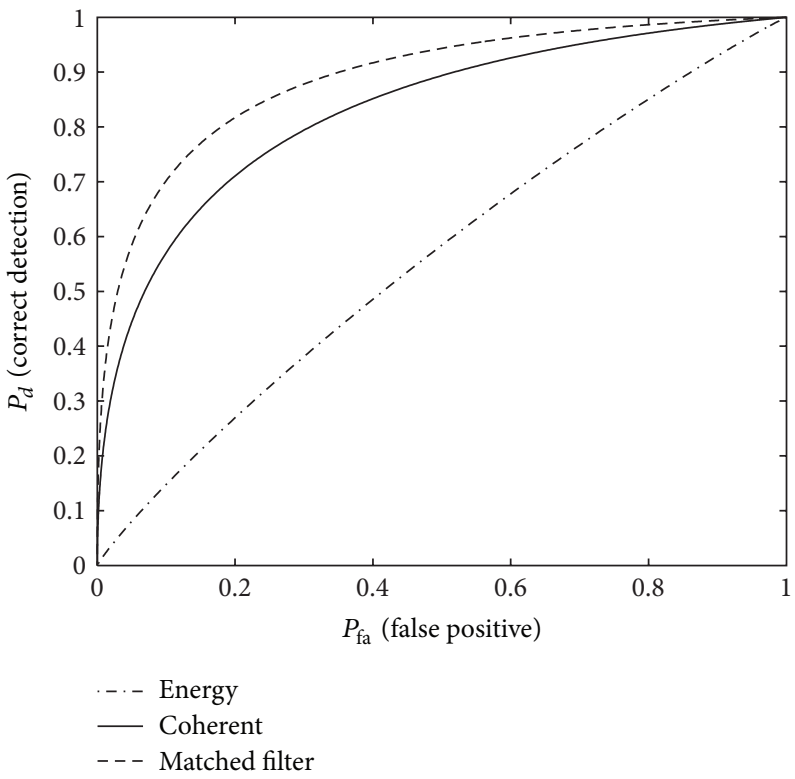

(d) ROC curve with 15 receivers

FIGURE 4: ROC curves for different number of receivers.

the approach in (27), comparing it to the conventional energy detector approach. It is not hard to show that

$$
f^{\prime}\left(y \mid H_{0}\right) \sim \text { Rayleigh }\left(\frac{\left\|p_{i}\right\| \sigma}{\sqrt{2}}\right)
$$

that is,

$$
p\left(f^{\prime}\right)=\frac{2 f^{\prime}}{\sigma^{2}} \exp \left[\frac{-\left(f^{\prime}\right)^{2}}{\sigma^{2}}\right] .
$$

The respective cdf is

$$
P\left(f^{\prime}\right)=1-\exp \left[\frac{-\left(f^{\prime}\right)^{2}}{\sigma^{2}}\right] .
$$

Similarly, it can be shown that

$$
f^{\prime}\left(y \mid H_{1}\right) \sim \operatorname{Rice}\left(\left|p_{i}^{H} p_{s}\right|, \frac{\left\|p_{i}\right\| \sigma}{\sqrt{2}}\right)
$$



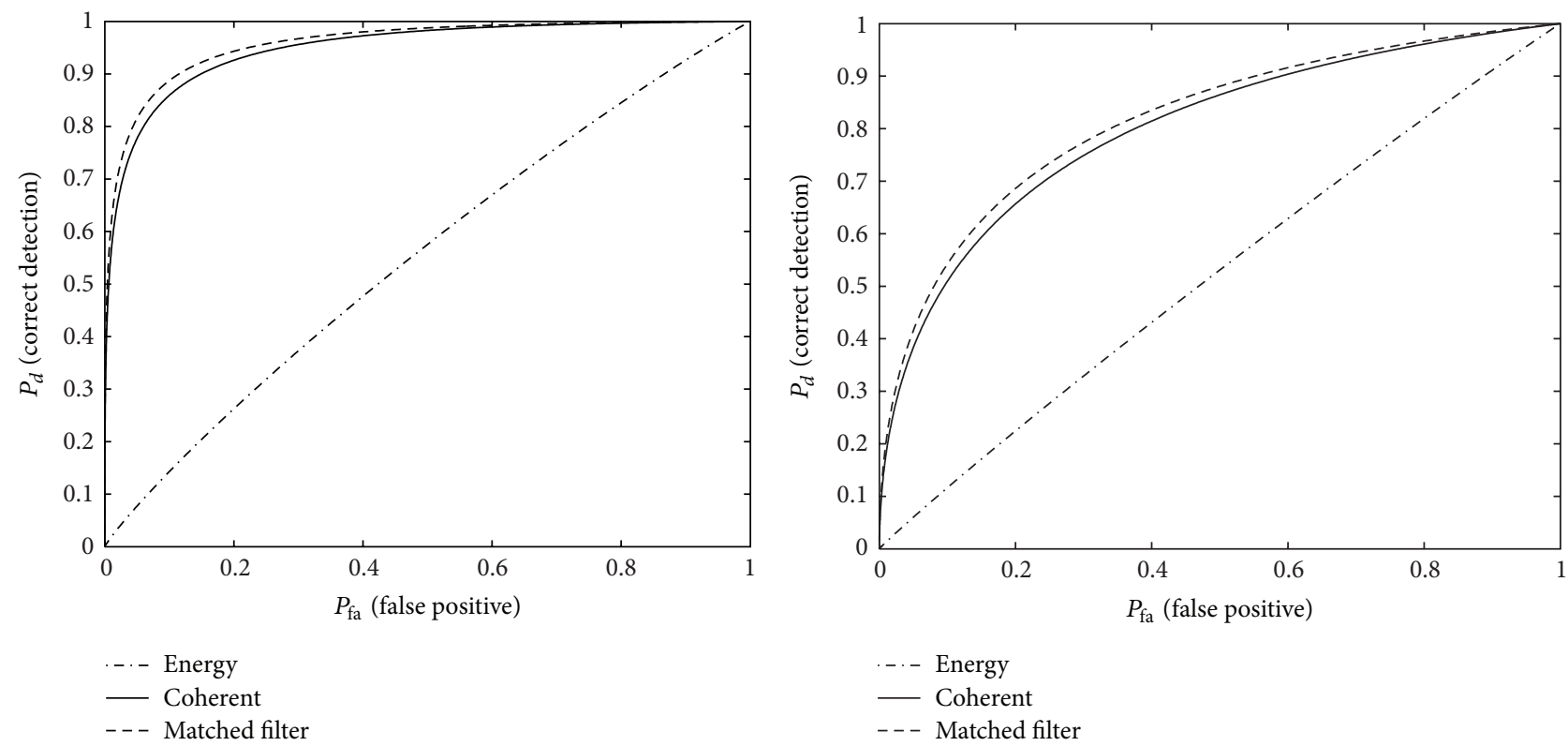

(b) ROC curve with SNR $=0.2$

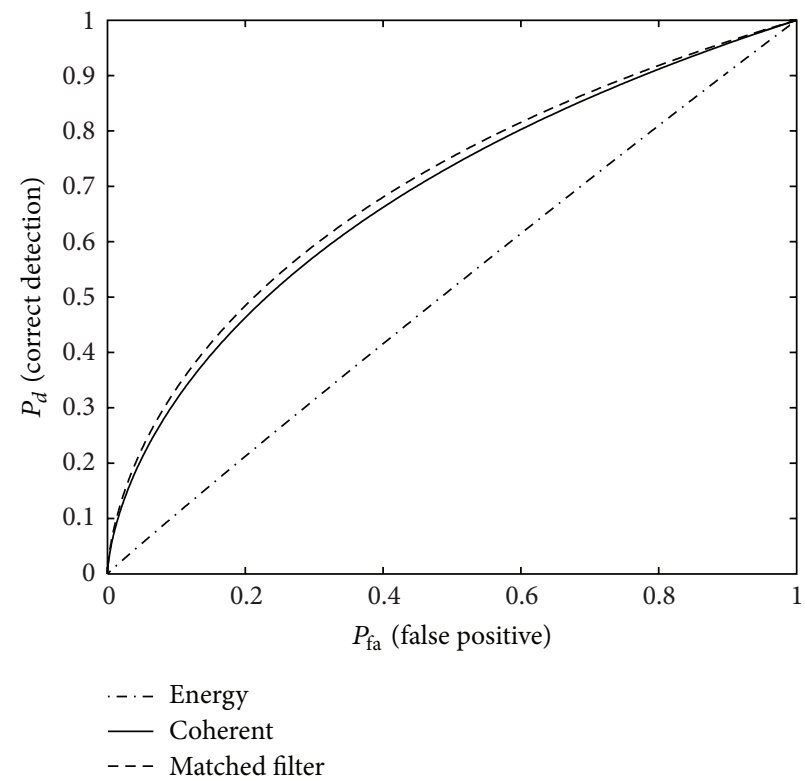

(c) ROC curve with SNR $=0.1$

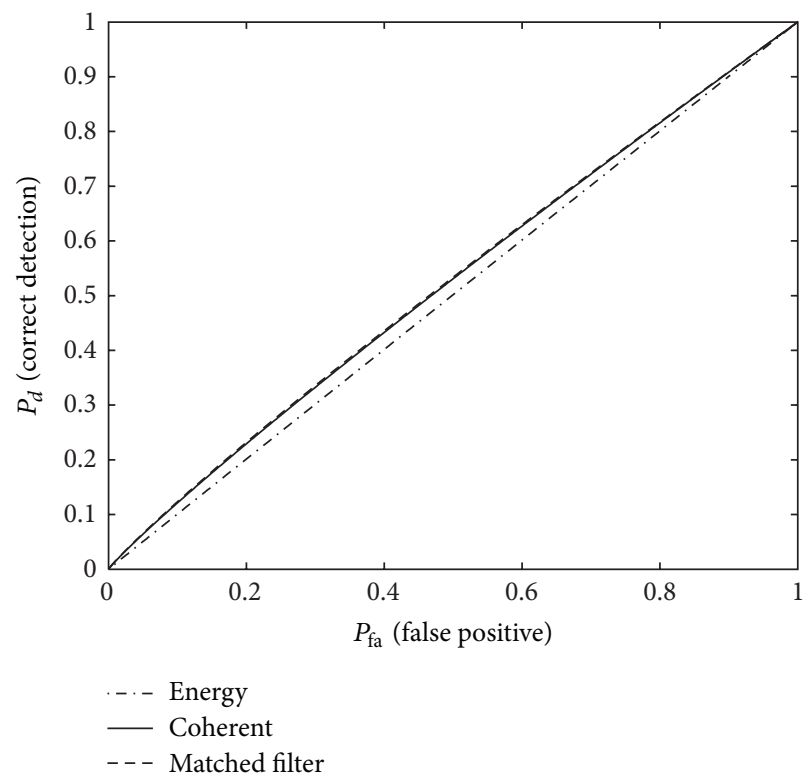

(d) ROC curve $\mathrm{SNR}=0.01$

FIGURE 5: ROC curves as a function of SNR.

that is,

$$
\begin{aligned}
p\left(f^{\prime}\right)= & \frac{2 f^{\prime}}{\left\|p_{i}\right\|^{2} \sigma^{2}} \exp \left\{\frac{-\left[\left(f^{\prime}\right)^{2}+\left|p_{i}^{H} p_{s}\right|^{2}\right]}{\left\|p_{i}\right\|^{2} \sigma^{2}}\right\} \\
& \times I_{0}\left(\frac{2 f^{\prime}\left|p_{i}^{H} p_{s}\right|}{\left\|p_{i}\right\|^{2} \sigma^{2}}\right),
\end{aligned}
$$

where $I_{0}$ is the modified Bessel function of the first kind with order zero. The respective cdf is

$$
P\left(f^{\prime}\right)=1-Q_{1}\left(\frac{\sqrt{2}\left|p_{i}^{H} p_{s}\right|}{\left\|p_{i}\right\| \sigma}, \frac{\sqrt{2} f^{\prime}}{\left\|p_{i}\right\| \sigma}\right),
$$

where $Q_{1}$ is the Marcum $Q$-function. 
It follows from (34) and (37) that for this detection approach with threshold $t$

$$
P_{d}=Q_{1}\left(\frac{\sqrt{2}\left|p_{i}^{H} p_{s}\right|}{\left\|p_{i}\right\| \sigma}, \frac{\sqrt{2} t}{\left\|p_{i}\right\| \sigma}\right)
$$

while

$$
P_{\mathrm{fa}}=\exp \left(\frac{-t^{2}}{\left\|p_{i}\right\|^{2} \sigma^{2}}\right) .
$$

This lays the foundation to analytically and numerically characterize the performance of the proposed coherent detection approach. Next we explain two numerical examples illustrating the proposed coherent detector. The first one corresponds to the detection of a small scatterer inside a cavity. The second one is the detection of a small scatterer in a medium formed by a large number of randomly positioned point scatterers. As required by the previous theory, the background media used in these examples are highly reverberating. Therefore, realistically sized time reversal mirrors can be used to synthesize the time-reversed version of the incident fields probing the scatterers.

\section{Numerical Simulation Results}

As a first example we consider the detection of a small perfectly reflecting scatterer in a waveguide of perfectly reflecting walls (see Figure 2). The system is two-dimensional (2D), approximating a system where one of the dimensions is much larger than the wavelength and there is no variation along that dimension, which allows treatment via the Helmholtz equation in $2 \mathrm{D}$ space. The excitation is due to a point source located far from the reflecting scatterer (not shown). The receiver array has $M$ elements. The field measured at the array without the reflecting scatterer corresponds to the background signal $p_{i}$. The field measured at the array with the scatterer corresponds to the scattered field signal $p_{s}$, which in the presence of realistic noise becomes the noisy scattered field signal $y$ as we explained before. In particular, we added white Gaussian noise with variance chosen to achieve a given signal-to-noise ratio (SNR) where we define SNR as

$$
\mathrm{SNR}=\frac{\left\|p_{s}\right\|^{2}}{M \sigma^{2}}
$$

where $\sigma^{2}$ is the noise variance.

Figure 3 shows, for 10 receivers, the histogram of $\bar{E}$ and $f^{\prime}$ with and without the target after 10000 realizations of noise where $\mathrm{SNR}=0.2$. The histogram has been normalized by the area in order to give an estimate of the probability distribution. Also shown in this plot in black lines are the analytical expressions for the probability distribution shown in expressions (21), (32), and (35), which match the empirical distribution almost perfectly.

The quality of a detector is determined by the socalled receiver operator characteristic (ROC) curve. It is constructed by plotting the correct detection (true positive) rate $P_{d}$ versus the false alarm (false positive) rate $P_{\mathrm{fa}}$. The

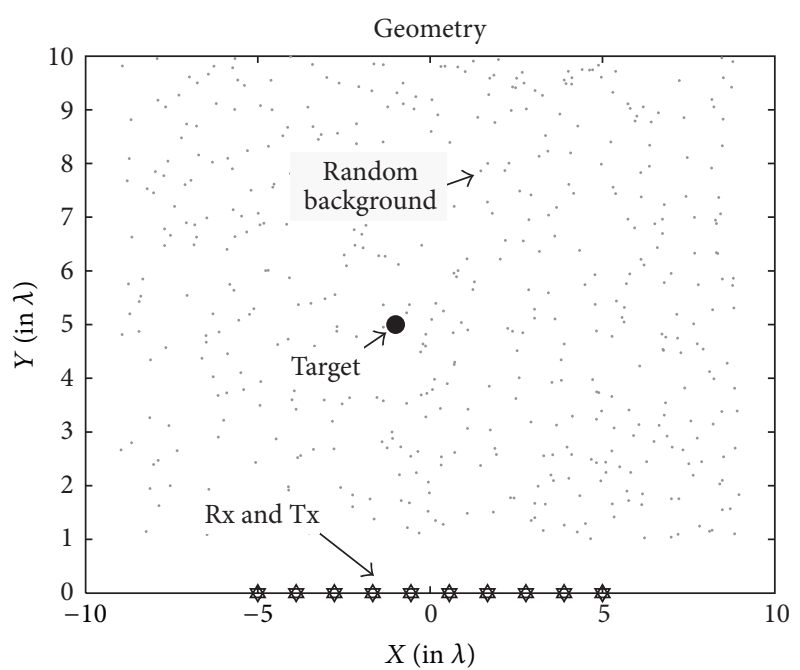

FIgURE 6: Geometry of the experiment, based on a collection of randomly positioned point scatterers forming the medium. The target is a point scatterer located inside this medium.

curve is created by assuming a large range of thresholds $t$ and calculating the corresponding correct detection and false alarm rates. In Section 3 we give the analytical expressions to calculate the probabilities of correct detection and false alarm. Figure 4 shows the ROC curves corresponding to an SNR of 0.2 and for different number of receivers. In the plots we compare the performance of the energy detector with the coherent one. For reference, we also show in these plots the ROC curves for the ideal detector, the matched filter, under the assumption that the background medium and the scatterer are known, and therefore the scattered signal is known (see (28) and discussion therein). The results consistently show that the new coherent detector is better than the conventional energy detector. The new coherent detector does not assume prior knowledge of the medium and scatterer, and therefore its performance cannot surpass that of the matched filter based on the scattered signal. This is confirmed in the plots. However, the new coherent detector performance is comparable to that of the matched filter which is based on full prior knowledge. The plots also show that for a small number of receivers (2-5) the coherent and energy detectors performed similarly but that as the number of receivers increases (10-15) the coherent detector performs considerably better than the energy detector, and, in fact, it approximates the matched filter receiver.

We also studied the effect of the SNR on the performance of the detectors. Figure 5 shows the ROC curves of the energy and coherent detectors for different SNR keeping the number of receivers at 10. The plots show that for very low SNR the performance of all detectors is similar. However, as we increase the SNR we start seeing a considerable advantage in using the coherent detector.

As a second example, we consider in $2 \mathrm{D}$ space a background system consisting of a collection of randomly positioned elastic point scatterers (see Figure 6). It is assumed that all the scatterers have the same scattering strength, but neither 


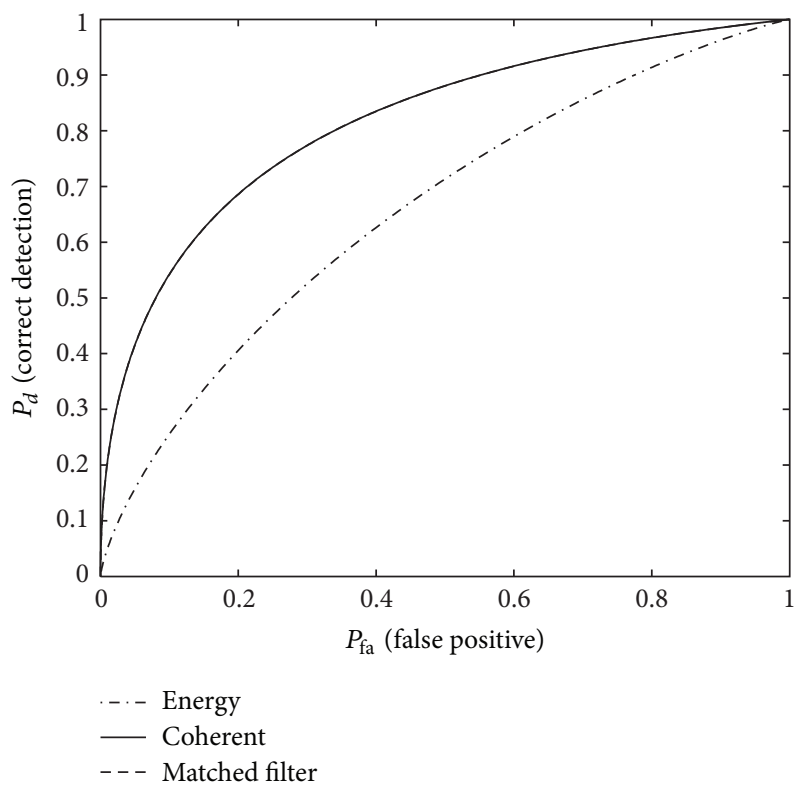

(a) Realization 1

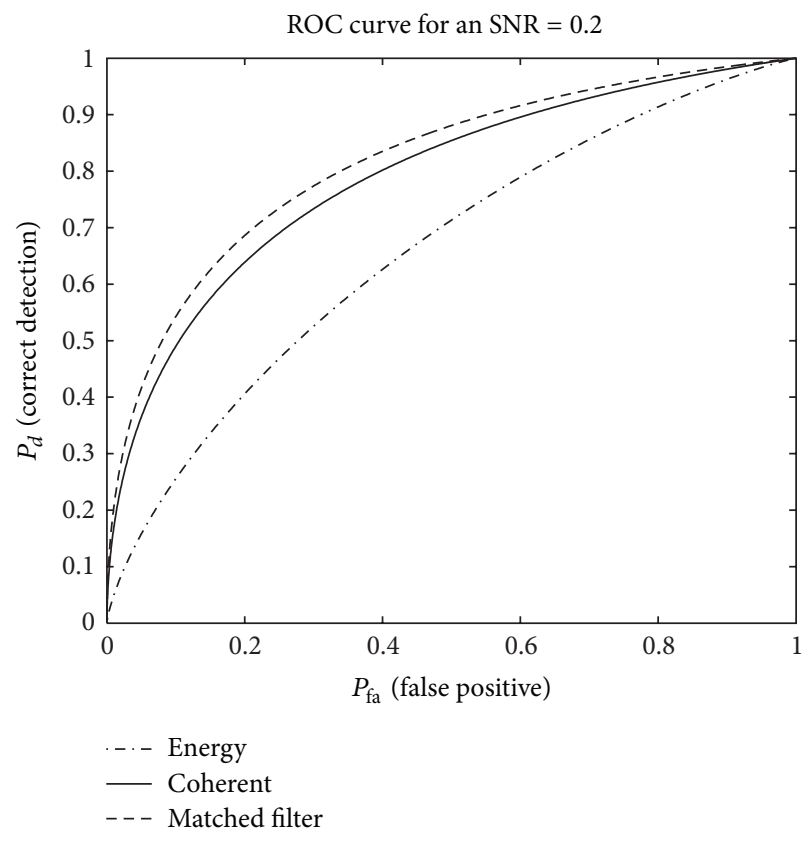

(c) Realization 3

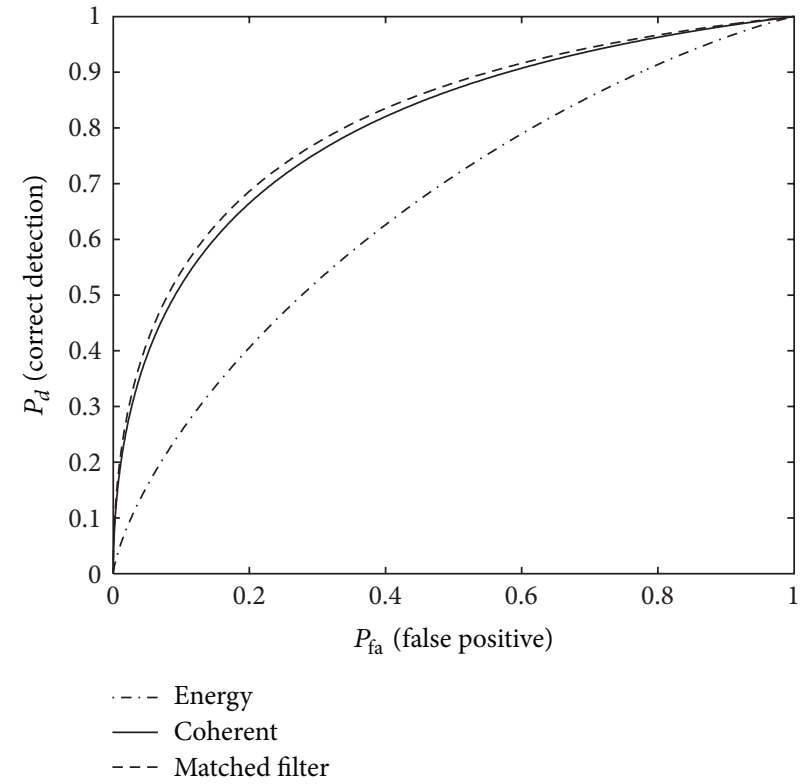

(b) Realization 2

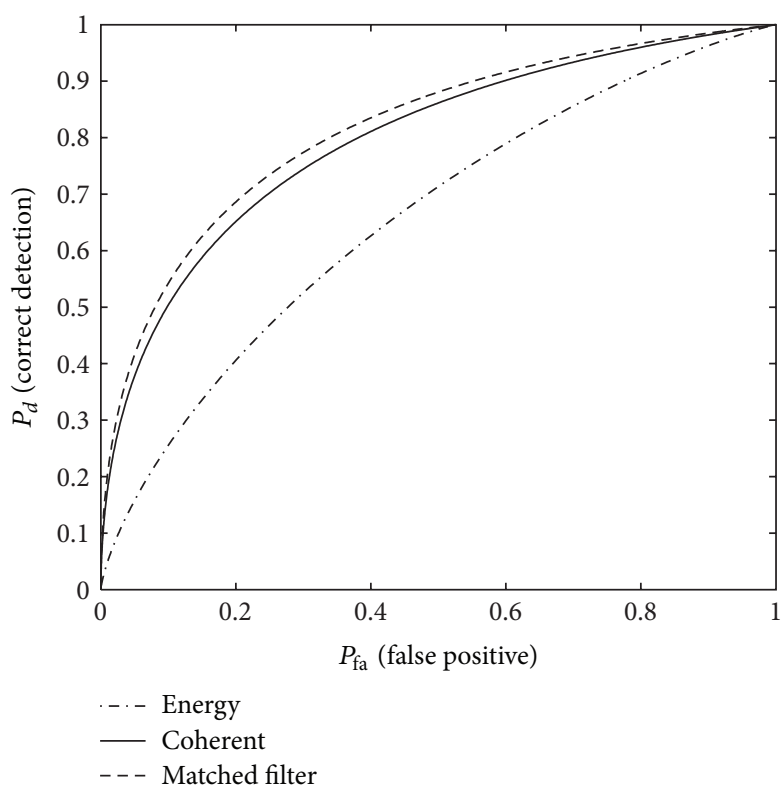

(d) Realization 4

FIGURE 7: ROC curves for different realizations of the random background.

the positions or strengths of these background scatterers are known during the implementation of the detection. The target has unit scattering strength and is positioned within the background. No prior knowledge of the target parameters is used in the detection process. The following results assume 10 receiver elements and $\mathrm{SNR}=0.2$.

Because of the random nature of the background, the performance of the detectors will vary depending on the location of the point scatterers, as shown in Figure 7. Overall the results are similar to what we found in the previous example. Thus the coherent detector outperforms the energy detector and has a performance that is close to that of the matched filter detector. Figure 8 shows the mean and standard deviation of the difference between the ROC curves of the coherent and energy detector showing the improvement in the detection performance when using the coherent approach over the energy approach. Also shown in the plot is the difference between the coherent detector and the matched filter which shows that their performance is very similar.

We conclude this section by addressing the effect of noise or perturbation of the incident, background signal over the performance of the proposed detection approach. 


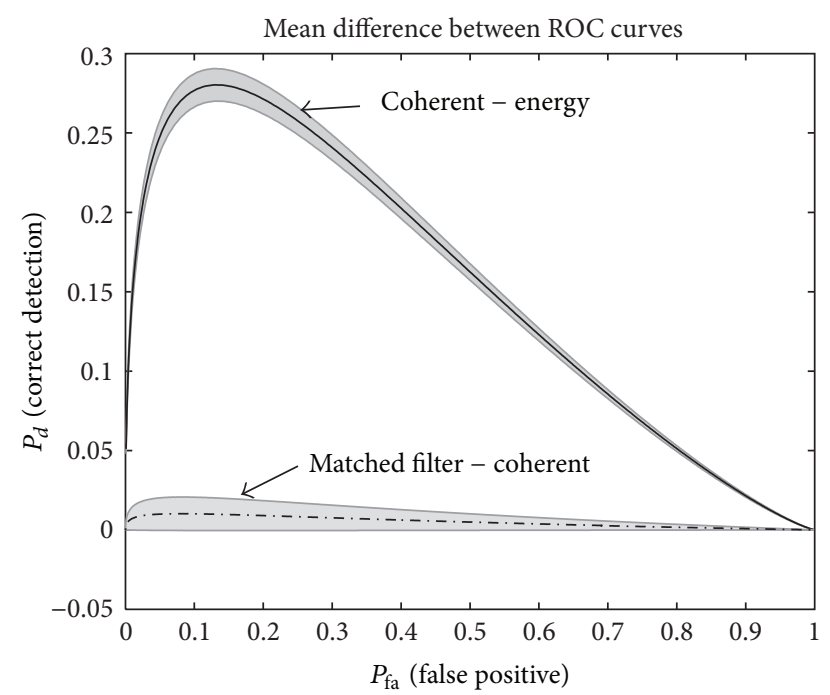

FIGURE 8: Difference in ROC curve between the coherent and energy detectors and between the matched filter and coherent detectors.

In particular, in the previous examples as well as in the expressions developed in Section 3 it was assumed that the incident field or background signal $\left(p_{i}\right)$ was measured with a high degree of accuracy. The analytical expressions for the probability of detection and false alarm were derived for the special case where the incident field signal was known perfectly. These expressions are no longer valid when the incident field signal is corrupted by significant noise. We consider next the effect of noisy incident fields on the performance of the coherent detector. We calculate the corresponding ROC curves by empirically estimating the rates of detection and false alarm from multiple experiments.

Figure 9 shows the ROC curves assuming 10 receivers, an SNR (in the scattered field signal) of 0.2 and different SNRs for the incident field signal. For very high SNR incident field signal the performance is similar to the previous experiments, that is, the performance of the coherent detector is significantly better than that of the energy detector and approximates the performance of the ideal matched filter. However, as we increase the level of noise in the incident field signal, the performance of the coherent detector starts decreasing to the point of performing worse than the energy detector. Therefore, for the envisioned applications, such as radar and surveillance, it will be necessary to implement first a learning phase in which a sufficiently large number of background signal samples are gathered, so as to obtain a high SNR background signal estimate to be used in subsequent detection decisions. We believe that this is feasible in some of the applications we have suggested so far. For example, in many practical security and military surveillance scenarios, for example, surveillance in a facility out of the regular business hours, the presence of an object of interest (a suspicious individual wandering in the facility) can be a rare event, which means that it is feasible to obtain and store a good background signal estimate and use it to implement the coherent detection method proposed in this work.

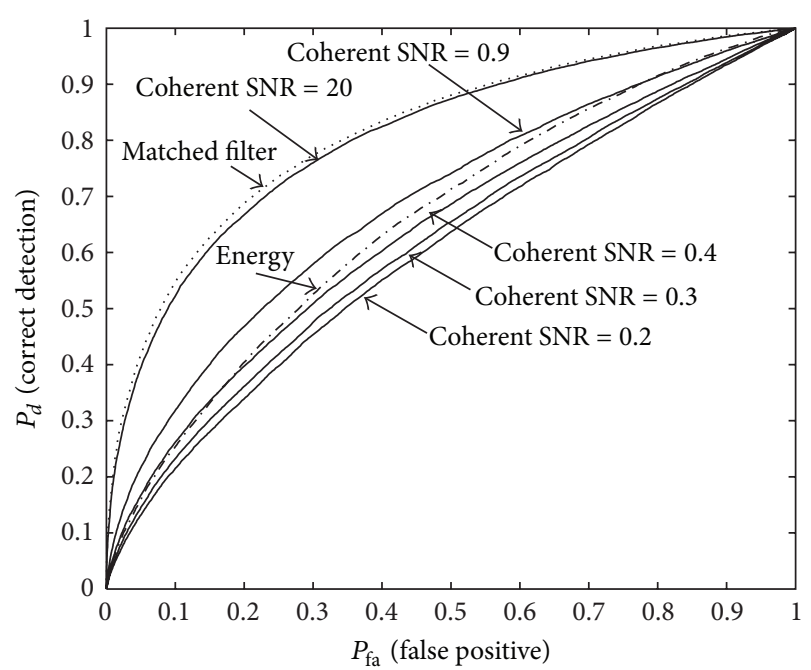

FIGURE 9: ROC curve for the matched filter detection, energy detector, and the coherent detector for different levels of SNR in the incident field. For all cases the SNR of the scattered field was kept fixed at 0.2 , and the number of receivers was kept at 10 .

\section{Conclusion}

We have presented the physical principles behind a new kind of coherent detector of unknown scatterers in unknown media. The proposed approach can work in highly reverberating media and with realistically sized sensing apertures, so long as they are large enough to function as time-reversal mirrors capable of approximately focusing the time reversal version of the incident fields probing the scatterer (via the time-reversal plus re-radiation steps associated to standard time reversal focusing). This approach thus has the potential of being very useful in target and change detection in complex environments relevant to safety and security, such as indoor facilities, caves, and tunnels, as well as the urban canyon. We laid the detection theory foundation to analytically and numerically characterize the new proposed detector and illustrated its feasibility with the help of numerical examples. The performance of the proposed coherent detection scheme was illustrated by means of ROC curves. The obtained results were compared to those of the standard incoherent energy detector and the optimal matched filter which assumes perfect knowledge of the target and the medium and is therefore only a reference performance bound (the matched filter is inapplicable under the realistic conditions in which one does not know the target or the medium). It was found that the coherent detector outperforms the energy detector as long as good (high SNR) estimates of the background signal are available. We found that, for certain realizations of the highly reverberating medium considered in the numerical study, the performance of the new coherent detector is surprisingly close to the optimal matched filter, despite the fact that (unlike the matched filter) the coherent detector assumes no knowledge whatsoever of the target and the medium. We also found that the performance of the coherent detection method decreases when the estimate of the background signal is 
significantly corrupted by noise. On the other hand, this is not a serious limitation in applications such as surveillance (of indoor facilities, etc.) where the presence of a target is a rare event so that it may be possible to get a good estimate of the background signal in the absence of a target. We plan to report elsewhere on the applications of the proposed coherent detection approach to surveillance and other fields.

\section{Acknowledgment}

This research was supported by the Air Force Office of Scientific Research Grant FA9550-12-1-0285.

\section{References}

[1] R. G. Newton, Scattering Theory of Waves and Particles, Springer, New York, NY, USA, 2nd edition, 1982.

[2] M. Born and E. Wolf, Principles of Optics, Cambridge University Press, Cambridge, UK, 7th edition, 2005.

[3] P. S. Carney, J. C. Schotland, and E. Wolf, "Generalized optical theorem for reflection, transmission, and extinction of power for scalar fields," Physical Review E, vol. 70, no. 3, Article ID 036611, 2004.

[4] D. R. Lytle II, P. S. Carney, J. C. Schotland, and E. Wolf, "Generalized optical theorem for reflection, transmission, and extinction of power for electromagnetic fields," Physical Review E, vol. 71, no. 5, Article ID 056610, 2005.

[5] E. A. Marengo, "A new theory of the generalized optical theorem in anisotropic media," IEEE Transactions on Antennas and Propagation, vol. 61, no. 4, pp. 2164-2179, 2013.

[6] M. Fink, "Time reversal of ultrasonic fields, part I: basic principles," IEEE Transactions on Ultrasonics, Ferroelectrics, and Frequency Control, vol. 39, no. 5, pp. 555-566, 1992.

[7] R. Carminati, R. Pierrat, J. de Rosny, and M. Fink, "Theory of the time reversal cavity for electromagnetic fields," Optics Letters, vol. 32, no. 21, pp. 3107-3109, 2007.

[8] S. Yon, M. Tanter, and M. Fink, "Sound focusing in rooms: the time-reversal approach," Journal of the Acoustical Society of America, vol. 113, no. 3, pp. 1533-1543, 2003.

[9] H. Urkowitz, "Energy detection of unknown deterministic signals," Proceedings of the IEEE, vol. 55, pp. 523-531, 1967.

[10] R. D. Hippenstiel, Detection Theory, CRC Press, Boca Raton, Fla, USA, 2001. 

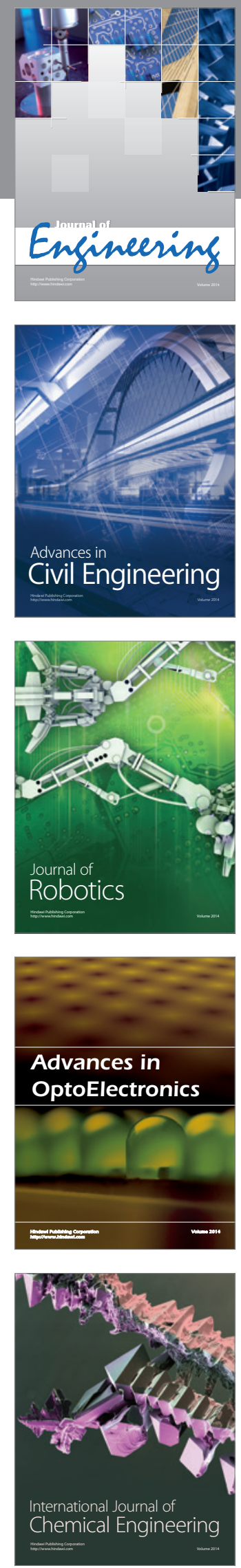

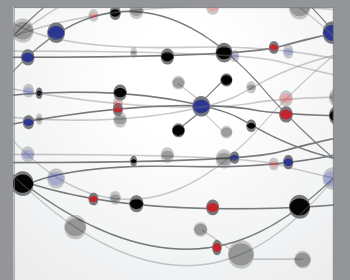

The Scientific World Journal
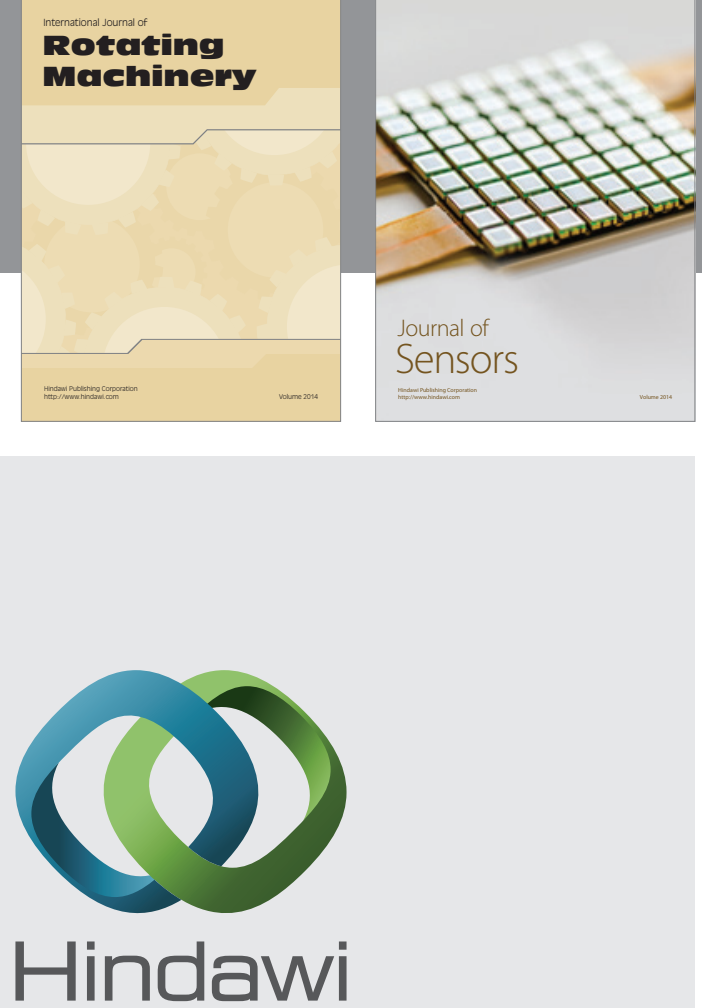

Submit your manuscripts at http://www.hindawi.com
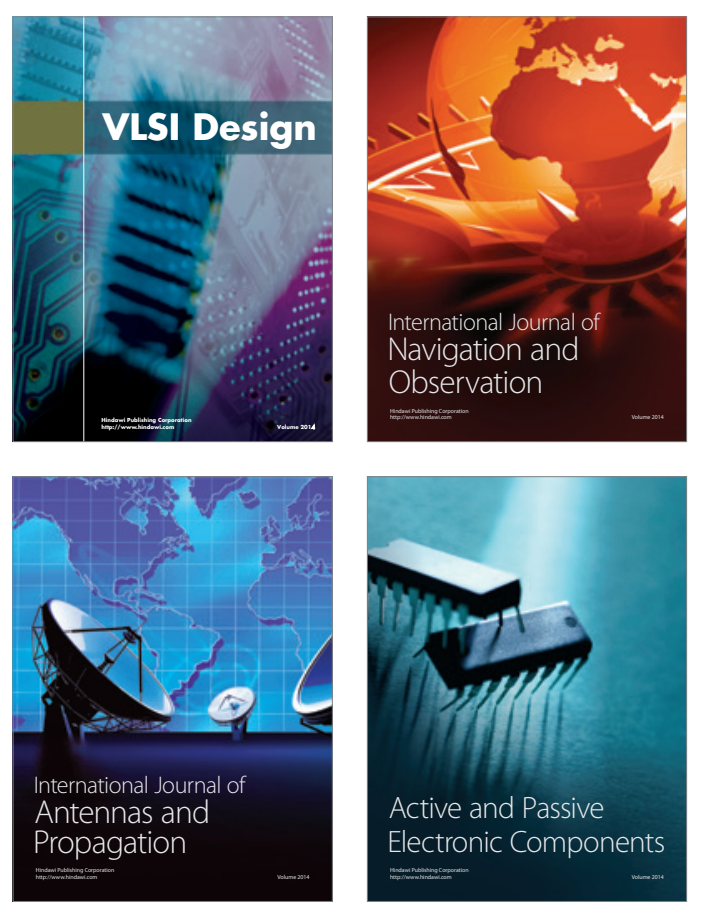
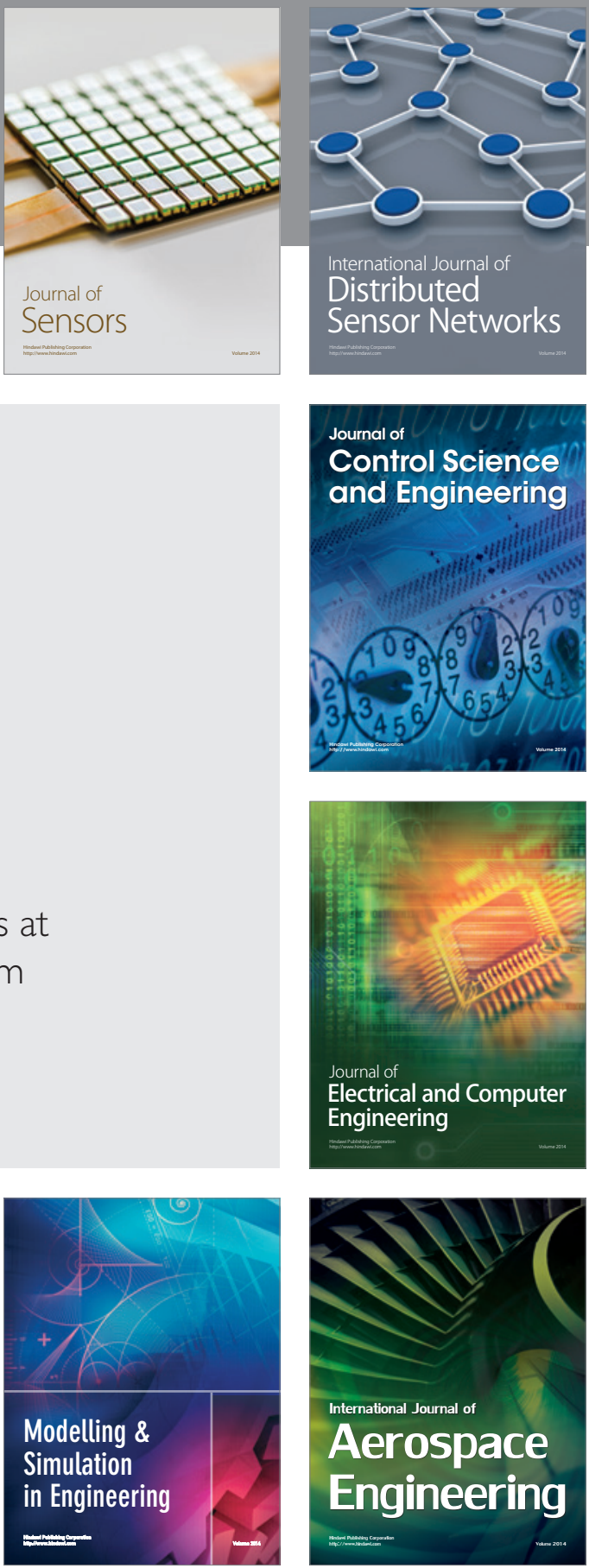

Journal of

Control Science

and Engineering
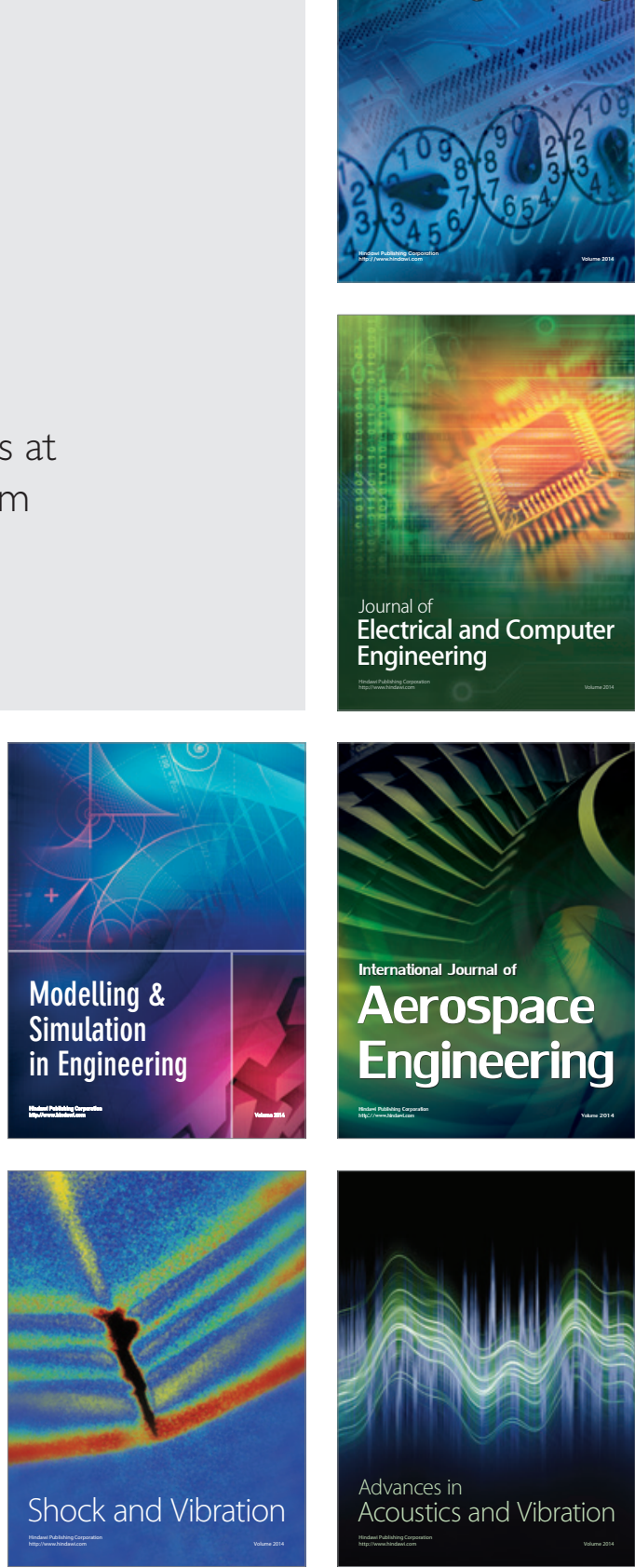